Intensivmed 2009 · 46:304-307

DOI 10.1007/s00390-009-0075-7

Online publiziert: 24. Juni 2009

(c) Springer-Verlag 2009

\author{
U. Janssens \\ Klinik für Innere Medizin, St.-Antonius-Hospital, Eschweiler
}

\section{Risikomanagement auf der Intensivstation}

Die politisch gewollten, massiven Veränderungen im ambulanten und stationären Sektor des Gesundheitswesens haben in den letzten Jahren sukzessiv sämtliche Bereiche der Medizin vor zum Teil unlösbar erscheinende Herausforderungen gestellt. Die Wirtschaftlichkeit der Medizin bestimmt unser Handeln immer mehr und hat eine Dimension erreicht, die inhaltliche, d. h. medizinische Probleme immer mehr in den Hintergrund treten lässt. Die Behandlung von Patienten orientiert sich nahezu ausschließlich an Vergütungsfragen. Der Behandlungsprozess wird entsprechend modifiziert, DRG-konform verkürzt und an zum Teil fragwürdige Evidenzen geknüpft. Eine sinnvolle, kosteneffektive und patientenzentrierte Medizin rückt derweil in immer weitere Ferne. Ein Kernproblem stellt sicherlich der undurchdringliche Dschungel der Partikularinteressen der verschiedenen Interessengruppen des Gesundheitssystems dar: Patienten, Ärzte, Apotheker, Verwaltungen, Krankenkassen, Politiker und die pharmazeutische Industrie verfolgen völlig unterschiedliche Ziele, die sich oftmals in ihrer Gegensätzlichkeit schlichtweg ausschließen.

Die Fortschritte der Medizin des 20. und 21. Jahrhunderts haben zu einer erheblichen Veränderung der Alterspyramide in den Industriegesellschaften geführt: Unsere Bevölkerung wird dank effizienter Diagnostik und Therapie immer älter aber sie wird eben nicht „gesund“ älter. Viele ältere Menschen sind von einer Reihe relevanter chronischer Erkrankungen betroffen, insbesondere sind hier kardiovaskuläre Erkrankungen wie die Herzin- suffizienz, Bluthochdruck und die koronare Herzkrankheit zu nennen, aber auch Diabetes mellitus, chronische Nierenfunktionseinschränkungen und neoplastische Erkrankungen finden sich häufig.

Die Prognose kritisch kranker Patienten ist eng mit dem Alter und den Komorbiditäten verknüpft. Dabei ist mit Prognose nicht nur das "nackte Überleben“ gemeint. Sondern auch andere - nicht nur gesundheitsökonomisch interessante - Endpunkte wie Intensiv- und Krankenhausliegedauer, langfristige Einschränkungen oder Behinderung und die (gesundheitsbezogene) Lebensqualität bzw. Lebenswertigkeit sind hiervon unmittelbar betroffen.

Im Jahr 2007 standen bei insgesamt 2087 Krankenhäusern in Deutschland 506.954 Betten für die stationäre Versorgung der Bevölkerung zur Verfügung, das entspricht 616 Betten/10o.ooo Einwohner (Quelle: Statistisches Bundesamt: Grunddaten der Krankenhäuser, Download 09.05.2009 http://www.gbe-bund.de). Von den 2087 Krankenhäusern halten 1286 Einrichtungen Betten zur intensivmedizinischen Versorgung vor $(61,6 \%)$, eine Fachabteilung „Intensivmedizin“ findet sich in 445 Krankenhäusern (21,3\%). Insgesamt sind 23.357 Betten für eine intensivmedizinische Versorgung designiert (4,6\% aller Krankenhausbetten). Im Jahr 2007 wurden 2.002.059 intensivmedizinische Behandlungsfälle erfasst. Dies entspricht bei 17.181.774 jährlichen stationären Behandlungsfällen einem Anteil von 11\%. Der Bedarf an Intensivbetten wird aufgrund der sich wandelnden demografischen Struktur der Gesellschaft unwei- gerlich wachsen. Damit werden auch die Aufgaben und Anforderungen an Ärzte, Schwestern und Strukturen in Akutkrankenhäusern größer.

\section{(7) Die hohen Kosten der Intensivmedizin bedingen entsprechende qualitative Anforderungen}

Intensivmedizin ist teuer und beansprucht einen überproportional hohen Anteil der Ressourcen der stationären Krankenversorgung und des Krankenhausbudgets: In den Vereinigten Staaten schwankt der Anteil der Kosten der Intensivmedizin an den gesamten Krankenhauskosten zwischen 11,5 und $13,3 \%$, das waren $0,56 \%$ des Bruttosozialproduktes [3]. Vergleichbare Zahlen liegen für Deutschland nicht vor und lassen sich bei Unkenntnis jeglicher Strukturdaten für die Gesamtheit der Intensivmedizin nicht zuverlässig abschätzen, auch wenn einzelne Intensivstationen in der Vergangenheit ihre individuellen Zahlen publiziert haben $[2,4,6,7]$. Andererseits ist eine Reihe von Interventionen, Operationen und Erkrankungen ohne die Hilfe der Intensivmedizin nicht behandelbar, sodass bei einer bereinigten Budgetbetrachtung diese Aspekte mit eingerechnet werden müssten. Neben den mittlerweile routinemäßig durchgeführten koronaren Bypass- und Herzklappenoperationen gilt dies z. B. auch für die intrakranielle Chirurgie, große Gefäßchirurgie oder endovaskuläre Interventionen an Herzklappen oder großen Gefäßen.

Gerade weil die Intensivmedizin verhältnismäßig hohe Kosten verursacht, er- 
Hier steht eine Anzeige.

算 Springer 


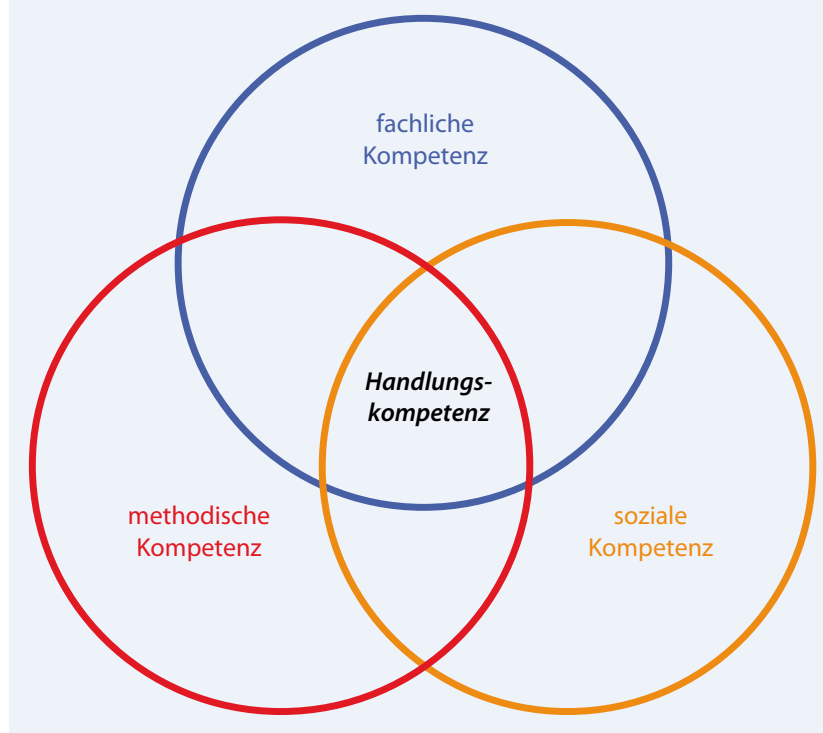

Abb. $1<$ Handlungskompetenz als Ergebnis einer methodischen, fachlichen und sozialen Kompetenz (freundlicherweise zur Verfügung gestellt von J. Graf) scheint es nachvollziehbar, dass entsprechende qualitative Anforderungen an diesen stationären Sektor gestellt werden. Dabei sollte unser Blick auf Aspekte der Struktur-, Prozess- und Ergebnisqualität gerichtet werden. Die Umsetzung wissenschaftlicher Erkenntnisse in die medizinische Praxis ist ein aufwendiger und multidimensionaler Prozess. Erstaunlicherweise unterscheiden sich Intensivstationen mit einer besseren Ergebnisqualität von Einrichtungen mit schlechteren Behandlungsergebnissen nur unwesentlich in ihren Strukturen. Die höhere Leistungsfähigkeit einzelner Intensivstationen ist im Wesentlichen von der Betriebskultur und den Eigenschaften der dort arbeitenden Menschen abhängig. Positiv auf die Behandlungsergebnisse wirken sich eine starke ärztliche und pflegerische Führung, patientenorientierte Arbeitsabläufe, gute Kommunikation und offene Konflikt- und Problemlösungsstrategien aus $[1,5,8,9]$.

Die hier vorliegende Ausgabe der "Intensivmedizin und Notfallmedizin" nähert sich dem Aspekt einer Verbesserung der Behandlungs- und Ergebnisqualität in der Intensivmedizin auf eine sicher nicht alltägliche Weise. Dennoch, gerade die hier dargestellten Themen besitzen eine fundamentale Bedeutung für unsere tägliche Arbeit, für unsere Patienten und für eine Verbesserung der Ergebnisqualität.

Selbstkritisch gefragt: Wer macht sich über die außerordentlich komplexen Entscheidungsabläufe in kritischen Situati- onen Gedanken und hat erkannt, dass gerade Schwierigkeiten in der Kommunikation diese Entscheidungsabläufe häufig genug behindern - ja, erfolgreiches Arbeiten sogar verhindern? Das Thema „Fehler, Behandlungsfehler, Irrtum“ ist mehr denn je auf den Titelseiten auch der renommierteren Tagespresse zu finden, doch wer der behandelnden Ärzte besitzt Kenntnisse über Ursachen von Behandlungsfehlern oder mögliche Vermeidungsstrategien? Täglich lesen wir, dass Ärzte Patienten fehlerhaft behandeln, Hygienemaßnahmen nur unzureichend treffen oder über Medikamentennebenwirkungen oder Arzneimittelinteraktionen wenig oder überhaupt nicht informiert sind.

Dieser zunehmende Druck von innen, aber auch von außen erzeugt Unsicherheit und auch Angst. Es wundert nicht, dass sich das ärztliche und nichtärztliche Personal immer wieder und immer häufiger in die innere Emigration zurückzieht: „Burn out“ ist kein populistisches Schlagwort, sondern stellt eine ernst zu nehmende Bedrohung für viele intensivmedizinisch tätige Ärzte und Pflegepersonen dar.

Beim Lesen der Beiträge von Andreas Valentin, Jürgen Graf, Marcus Rall und Wilhelm Kantner-Rumplmair wird der Leser sicher kleinere thematische Überschneidungen feststellen, aber dadurch eben auch erkennen, wie eng diese Themen miteinander verknüpft sind und welche praktische Relevanz sie für uns und unsere Arbeit besitzen.
Wir Intensivmediziner müssen uns mehr denn je solchen Themen zuwenden und unser Denken und Handeln auch auf diese eigentlich medizinfernen Themen fokussieren. Nur so werden wir uns einer optimalen Medizin, d. h. bestmöglichen Patientenbetreuung, annähern. "Soft Skills" werden nicht vererbt, sondern müssen hart erarbeitet und am Leben gehalten werden. Die (Intensiv-)Medizin benötigt dringend eine (neue) Kultur des Umgangs: Disziplin, Umgangsformen, Höflichkeit, Freundlichkeit, Motivation, sprachliche Kompetenz, Selbstständigkeit, Respekt und Teamfähigkeit dürfen keine Schlagworte bleiben, sondern müssen eine Kernkompetenz unseres ärztlichen Handelns darstellen (• Abb. 1).

Der aufmerksame Leser wird dieses erkennen und akzeptieren; sich aber frustriert abwenden, da bis zum heutigen Tage und auch für die Zukunft nicht erkennbar ist, wie angesichts fehlender oder völlig unzureichender personeller und struktureller Voraussetzungen eine gesunde und effiziente Betriebs-, Kommunikationsund Fehlerkultur auf unseren Intensivstationen etabliert werden soll. Um die in den Beiträgen genannten Vorschläge und Strategien umsetzen zu können, benötigen wir Zeit und Menschen - von beiden haben wir (nachweislich!) zu wenig.

\section{(2) Das G-DRG-System bildet Maßnahmen zur Qualitätsver- besserung unzureichend $a b$}

Aus meiner Sicht müssen die Führungskräfte, aber auch die medizinischen Fachgesellschaften gemeinsam mit den Administrationen bei Krankenkassen und Gesundheitspolitikern auf dieses enorme und nicht aus den vorhandenen Ressourcen zu deckende Defizit nachdrücklich hinweisen. Das Institut für das Entgeltsystem im Krankenhaus (InEK) entwickelt kontinuierlich das G-DRG-System, basierend auf den Kosten- und Leistungsdaten einer Stichprobe deutscher Krankenhäuser, fort. Die Kostendaten werden dabei im Rahmen eines Ist-KostenAnsatzes auf Vollkostenbasis anhand der Vorschriften des Kalkulationshandbuches in den an der Kalkulation teilnehmenden Krankenhäusern einheitlich ermittelt 
(www.g-drg.de, Zugriff 10.05.2009). Im augenblicklichen Entgeltsystem sind Maßnahmen zur Qualitätsverbesserung, Schulung, Aus- und Weiterbildung völlig unzureichend abgebildet. In den meisten Krankenhäusern werden Fehlerberichtssysteme - so überhaupt vorhanden - und die damit verbundenen personellen Ressourcen kostenneutral entwickelt und implementiert.

Andererseits dürfen wir uns angesichts der derzeitigen Situation nicht entmutigen lassen, sondern sollten die Erkenntnisse aufgreifen und in kleinen Schritten voranbringen. Unsere Patienten werden es uns danken.

„Der Begriff, Fortschritt' allein setzt bereits die Horizontale voraus. Er bedeutet ein Weiterkommen und kein Höherkommen."

Joseph Roth (1894-1939), österreichischer Schriftsteller und Journalist

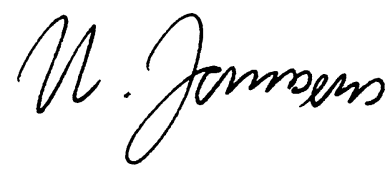

U. Janssens

\section{Korrespondenzadresse}

\section{Prof. Dr. U. Janssens}

Klinik für Innere Medizin, St.-Antonius-Hospital Dechant-Deckers-Str. 8, 52249 Eschweiler uwe.janssens@sah-eschweiler.de

Interessenkonflikt. Der korrespondierende Autor gibt an, dass kein Interessenkonflikt besteht.

\section{Literatur}

1. Graf J (2007) Qualitätsmanagement in der Intensivmedizin. Habilitationsschrift. Philipps-Universität, Marburg

2. Graf J, Graf C, Janssens U (2002) Analysis of resource use and cost-generating factors in a German medical intensive care unit employing the Therapeutic Intervention Scoring System (TISS-28). Intensive Care Med 28:324-331

3. Halpern NA, Pastores SM, Greenstein RJ (2004) Critical care medicine in the United States 19852000: an analysis of bed numbers, use and costs. Crit Care Med 32:1254-1259

4. Klepzig H, Winten G, Thierolf C et al (1998) Behandlungskosten auf einer medizinischen Intensivstation. Dtsch Med Wochenschr 123:719-725

5. Luce JM (1991) Improving the quality and utilization of critical care. QRB Qual Rev Bull 17:42-47
6. Moerer O, Plock E, Mgbor U et al (2007) A German national prevalence study on the cost of intensive care: an evaluation from 51 intensive care units. Crit Care 11:R69

7. Moerer O, Schmid A, Hofmann M et al (2002) Direct costs of severe sepsis in three German intensive care units based on retrospective electronic patient record analysis of resource use. Intensive Care Med 28:1440-1446

8. Shortell SM, Zimmerman JE, Rousseau DM et al (1994) The performance of intensive care units: does good management make a difference? Med Care 32:508-525

9. Zimmerman JE, Shortell SM, Rousseau DM et al (1993) Improving intensive care: observations based on organizational case studies in nine intensive care units: a prospective, multicenter study. Crit Care Med 21:1443-1451

\section{Abbott schreibt Dr.-Ernst- Wiethoff-Preis für innovative klinische Forschung aus}

Das Gesundheitsunternehmen Abbott schreibt zum siebten Mal in Deutschland den Dr. Ernst Wiethoff-Preis aus. Ausgezeichnet wird eine innovative Originalarbeit im Bereich der klinisch orientierten medizinischen Forschung aus folgenden Disziplinen: Innere Medizin, Rheumatologie, Gastroenterologie, Immunologie, Infektiologie, Pädiatrie, Dermatologie, Anästhesie und Intensivmedizin. Auch Arbeiten aus benachbarten Bereichen, die diese Fachrichtungen berühren, sind zugelassen. Bewerbungsfrist für die Einreichungen ist der 31. Dezember 2009. Der Preis geht zurück auf den ehemaligen Medizinischen Direktor von Abbott Deutschland, Dr. med. Ernst Otto Wiethoff. Das Preisgeld beträgt wie in den Vorjahren EUR 25.000,--

Für die Verleihung des Preises können sich Kandidaten bewerben, deren Arbeiten von einer renommierten Fachzeitschrift in den Jahren 2008 oder 2009 publiziert oder bis Jahresende 2009 akzeptiert wurden. Sollten sich mehrere Autoren aus einer Autorengruppe bewerben, ist bei Einreichung zu erklären, wer sich als Repräsentant der Gruppe bewirbt. Der Bewerber muss weiterhin anzeigen, ob er sich mit dieser Arbeit bereits für andere Preise beworben hat. Er muss zum Zeitpunkt der Fertigstellung der Arbeit an einem deutschen Forschungsinstitut beschäftigt oder in dessen Auftrag bzw. im Auftrag einer deutschen Forschungsfördereinrichtung im Ausland tätig sein. Das Höchstalter der Bewerber beträgt 38 Jahre. Die Begutachtung der eingereichten Arbeiten erfolgt durch ein unabhängiges, interdisziplinäres Gremium universitärer Professoren.

Bewerbungen mit Originalarbeit, Curriculum vitae und Publikationsverzeichnis sind in einfacher Ausführung spätestens bis zum 31. Dezember 2009 an Abbott zu richten.

Anschrift:

PD Dr. med. Johannes Zahner Medizinischer Direktor Abbott GmbH \& Co. KG Max-Planck-Ring 2 65205 Wiesbaden Tel. 06122-581178 